Draft version OCtober 29, 2018

Preprint typeset using $\mathrm{LAT}_{\mathrm{E}} \mathrm{X}$ style emulateapj v. 11/10/09

\title{
ENERGY SPECTRUM AND CHEMICAL COMPOSITION OF ULTRA-HIGH ENERGY COSMIC RAYS FROM SEMI-RELATIVISTIC HYPERNOVAE
}

\author{
RuO-Yu LiU ${ }^{1,2}$ AND XiAng-YU WANG ${ }^{1,2}$ \\ Draft version October 29, 2018
}

\begin{abstract}
It has been suggested that hypernova remnants, with a substantial amount of energy in semirelativistic ejecta, can accelerate intermediate mass or heavy nuclei to ultra-high energies and provide sufficient amount of energy in cosmic rays to account for the observed flux. We here calculate the expected energy spectrum and chemical composition of ultra-high energy cosmic rays from such semirelativistic hypernovae. With a chemical composition equal to that of the hypernova ejecta and a flat or hard spectrum for cosmic rays at the sources, the spectrum and composition of the propagated cosmic rays observed at the Earth can be compatible with the measurements by the Pierre Auger Observatory.
\end{abstract}

Subject headings: cosmic rays - gamma rays: bursts

\section{INTRODUCTION}

Ultra high energy cosmic rays (UHECRs) are the most energetic particles ever detected in the universe. There is a consensus that UHECRs with energies larger than $10^{19} \mathrm{eV}$ are of extra-galactic origin. However, the composition of ultra high energy cosmic rays at these energies remains disputed. Recent observations of their maximum air shower elongations $<\mathrm{X}_{\max }>$ and fluctuations $\operatorname{RMS}\left(<\mathrm{X}_{\max }>\right)$ by the Pierre Auger Observatory (PAO) suggest that UHECRs are progressively dominated by heavier nuclei at energies above a few EeV (Abraham et al. 2010), while the results from High Resolution Fly's Eye Experiment (HiRes) and Telescope Array (TA) are consistent with a pure proton composition (Abbasi et al. 2004; Tsunesada et al. 2011). These claims, however, depend on the poorly-understood hadronic interaction models at such high energies. Nevertheless, the advantage of heavy composition for UHECRs is that heavy or intermediate-mass particles are much easier to be accelerated to UHE energies than protons as the maximum particle energy is rigidity dependent (i.e. proportional to the nuclear charge $Z$ of the particles). Heavy nuclei with energy above $100 \mathrm{EeV}$ will suffer from strong photo-disintegration attenuation due to interactions with cosmic microwave background $(\mathrm{CMB})$ and cosmic infrared background (CIB) photons, with an attenuation length shorter than $\sim 100 \mathrm{Mpc}$ (see e.g. Puget et al. 1976; Allard et al. 2006; Hooper et al. 2007; Allard 2009; Aloisio 2011; Taylor et al. 2011). Thus, sources producing UHECRs above $100 \mathrm{EeV}$ must be within $\sim 100 \mathrm{Mpc}$. Within this distance, there are few sources that are powerful enough to be able to accelerate particles to energies $10^{20} \mathrm{eV}$. The candidates include local active galactic nuclei (AGNs) (e.g. Biermann \& Strittmatter 1987; Berezinsky et al. 2006; Pe'er et al. 2009) and gamma-ray bursts (GRBs) (e.g. Waxman 1995, 2004; Vietri 1995; Wick et al. 2004; Dermer

\footnotetext{
${ }^{1}$ Department of Astronomy, Nanjing University, Nanjing, 210093, China

${ }^{2}$ Key laboratory of Modern Astronomy and Astrophysics (Nanjing University), Ministry of Education, Nanjing 210093, China
}

\& Atoyan 2006; Murase \& Nagataki 2006; Murase et al. 2006).

Extragalactic hypernova has been suggested to be a candidate source of UHECRs as well(Wang et al. 2007, 2008; Liu et al. 2011). Hypernovae are a peculiar type of supernovae that are more bright and have larger explosion energies than typical ones (Paczyński 1998; Soderberg et al. 2006). Several hypernovae have been observed in the nearby universe to date, usually associated with sub-energetic GRBs. For example, SN 1998bw, associated with GRB 980425, is the first-found peculiar supernova at a distance of $38 \mathrm{Mpc}$, with an isotropicequivalent total kinetic energy of $\sim 5 \times 10^{52} \mathrm{erg}$ (Galama et al. 1998; Kulkarni et al. 1998; Iwamoto et al. 1998). Radio afterglow of this event showed that more than $10^{50}$ erg of kinetic energy was released in the form of a mildly relativistic ejecta. SN 2006aj, associated with GRB 060218, was detected at a distance of $140 \mathrm{Mpc}$ (Campana et al. 2006; Mirabal et al. 2006; Soderberg et al. 2006; Mazzali et al. 2006). The strong thermal Xray emission component in GRB 060218 is interpreted as arising from a semi-relativistic supernova shock breakout, in which a mildly relativistic ejecta with energy about $10^{50}$ erg has been inferred (Campana et al. 2006; Soderberg et al. 2006). Radio observations of the recently discovered supernova SN2009bb, for which no associated GRB is found, also suggest that more than $10^{50} \mathrm{erg}$ is coupled into the mildly-relativistic ejecta(Soderberg et al. 2010). We will use the term semi-relativistic hypernovae to denote such supernovae harbouring mildly relativistic ejecta. Such semi-relativistic ejecta drives semi-relativistic shocks, which could accelerate particles to ultra-high energies (Wang et al. 2007). The recently proposed engine-driven supernova as a source for UHECRs are identical to the hypernovae in nature (Ray \& Chakraborti 2011), since both are powered by the central engine and both have much more energy in semi-relativistic ejecta. The estimated event rate of SN2009bb-like engine-driven supernovae is also comparable to the hypernova rate(Soderberg et al. 2010). The progenitors of hypernovae are believed to be Wolf-Rayet (WR) stars, which are stripped of their original $\mathrm{H}$-rich 
and sometimes even He-rich envelopes. The circumstellar winds are therefore rich in intermediate mass elements, such as $\mathrm{C}$ and $\mathrm{O}$. Moreover, the hypernovae ejecta contain heavy elements such as Fe that are produced by nuclear fusion in the core of exploding WR stars, as well as elements heavier than Fe that are synthesized during the explosions.

In this paper, we study the propagated energy spectrum and chemical composition of UHECRs that originate from hypernovae, and confront them with the measured data by PAO. The rest of this paper is organized as follows. In $\S 2$, we first briefly review the hypernova scenario and describe the source composition of UHECRs produced by hypernovae. In $\S 3$, we calculate the energy spectrum and composition of UHECRs arriving at the Earth after propagation and then confront them with the Pierre Auger observations. Finally, we give our discussions and conclusions in $\S 4$. The effect of intergalactic magnetic fields is neglected in this paper. Throughout the paper, we use $\mathrm{eV}$ as the unit of particle energy and use c.g.s units for other quantities and denote by $Q_{x}$ the value of the quantity $Q$ in units of $10^{x}$.

\section{THE CHEMICAL COMPOSITION AND ENERGY SPECTRUM OF UHECRS AT THE SOURCE}

\subsection{Hypernova model for UHECRs}

Wang et al. $(2007,2008)$ have suggested that hypernova remnants can accelerate heavy or intermediate mass nuclei to ultrahigh energies above $10^{19} \mathrm{eV}$. Here for simplicity we only focus on the semi-relativistic part of the ejecta, since only this part is relevant to the acceleration of UHECRs. Particles are accelerated in the shock region where the semi-relativistic ejecta is freely expanding before being decelerated by the swept-up circum-stellar medium. The size of this free-expansion phase region for ejecta of a particular velocity $\beta_{\mathrm{sh}} c$ and kinetic energy $E_{K}$ is $R_{\mathrm{HN}}=E_{k} v_{w} / \Gamma^{2} c^{2} \dot{M} \simeq 2 \times 10^{17} E_{k, 51} \Gamma^{-2} \dot{M}_{-5}^{-1} v_{w, 3} \mathrm{~cm}$, where $\Gamma=\left(1-\beta_{\mathrm{sh}}^{2}\right)^{-1 / 2}$ is the bulk Lorentz factor of the ejecta, $\dot{M}=10^{-5} \dot{M}_{-5} \mathrm{M}_{\odot} \mathrm{yr}^{-1}$ is the wind mass loss rate (whose average value is $3 \times 10^{-5} \mathrm{M}_{\odot} \mathrm{yr}^{-1}$ for WR stars), and $v_{w}=10^{3} v_{w, 3} \mathrm{kms}^{-1}$ is the wind velocity (Willis 1991; Chevalier \& Li 1999). We assume a fraction of $\epsilon_{B}$ of shock internal energy goes into the magnetic field and hence the magnetic field energy density is $B^{2} / 8 \pi \approx 2 \Gamma^{2} \beta^{2} \epsilon_{B} \rho_{w} c^{2}$, where $\rho_{w}=\dot{M} / 4 \pi R_{\mathrm{HE}}^{2} v_{w}$ is the mass density of the stellar wind at radius $R_{\mathrm{HN}}$. Thus, by equating the adiabatic cooling time with the acceleration time, the maximum energy of accelerated particles is

$$
\begin{aligned}
E_{\max } & \simeq Z e \Gamma B R_{\mathrm{HE}} \beta_{\mathrm{sh}} \\
& =3.7 \times 10^{20}\left(\frac{Z}{26}\right) \epsilon_{B,-1}^{1 / 2} \Gamma^{2} \beta_{\mathrm{sh}}^{2} \dot{M}_{-5}^{1 / 2} v_{w, 3}^{-1 / 2} \mathrm{eV} .
\end{aligned}
$$

where $Z$ is the nuclear charge number of the particle and $e$ is the charge of electrons. Note that, in the semirelativistic hypernova scenario, the adiabatic cooling of UHE nuclei is much more efficient than other cooling processes, such as synchrotron cooling, photopion production and photo-disintegration processes (Wang et al. 2008), so we do not consider them here.

Hypernova can also provide sufficient energy in UHECRs(Wang et al. 2007; Liu et al. 2011). According to
Katz et al. (2009), the energy production rate ${ }^{3}$ in UHECRs per logarithmic energy interval inferred from the measured flux by PAO is $\sim 10^{43.5} \mathrm{ergMpc}^{-3} \mathrm{yr}^{-1}$. Hypernova ejecta with $\Gamma \beta \sim 0.5$ can produce cosmic rays with energies $\sim 10^{20} \mathrm{eV}$ and the kinetic energy in such velocity ejecta is typically $\sim 10^{51} \mathrm{erg}$ (e.g. Wang et al. 2007; Chakraborti et al. 2011). Assuming that half of the kinetic energy goes into cosmic rays and that $10 \%$ of this energy is distributed in every logarithmic energy interval, the required local event rate of hypernovae is then $\sim 600 \mathrm{Gpc}^{-3} \mathrm{yr}^{-1}$, which is consistent with the observed rate (e.g. Soderberg et al. 2006; Liang et al. 2007).

\subsection{Composition and spectrum at the sources}

It is usually assumed that shock acceleration leads to a power-law energy spectrum for particles. The differential number of particles accelerated by one hypernova can be described by

$$
\frac{d N_{i}}{d E_{i}}=\frac{U_{i} E_{i}^{-p}}{\int_{E_{i, \min }}^{E_{i, \max }} d E_{i} E_{i}^{1-p}} \exp \left(-\frac{E_{i}}{E_{i, \max }}\right) \exp \left(-\frac{E_{i, \min }}{E_{i}}\right)
$$

where the subscription $i$ represents the species of the nuclei (e.g. $i=\mathrm{H}, \mathrm{He}$, and etc.), $E_{i, \max }$ and $E_{i \text {,min }}$ are respectively the maximum and minimum energy of cosmic rays injected into the intergalactic space by the hypernova, and $U_{i}$ is the total energy of nuclei of species $i$ released by one hypernova into the intergalactic space. According to Eq. 1, hypernova remnants can accelerate iron nuclei to energies about $\sim 5 \times 10^{20} \mathrm{eV}$, so we fix $E_{\max , \mathrm{Fe}}=10^{20.5}(Z / 26) \mathrm{eV}$ in the following calculation. $E_{i, \text { min }}$ is the low energy cutoff in the spectrum, since nuclei with energy below $E_{i, \min } \approx Z_{i} e B_{\text {host }} R_{\text {host }}$ can not escape from the confinement by the magnetic fields in the host galaxies, where $B_{\text {host }}$ is the typical magnetic field of the host galaxy and $R_{\text {host }}$ is the scale height of the host galaxy. Taking our own galaxy as an approximation, we have $E_{\min , \mathrm{Fe}} \approx 10^{19}(Z / 26) B_{-6}\left(h_{z} / 0.3 \mathrm{kpc}\right) \mathrm{eV}$, where $h_{z}$ is the scale height of the Galaxy. For a single velocity ejecta ${ }^{4}$, the power-law index is usually assumed to be $p \simeq 2$ for both non-relativistic shocks and semi-relativistic shocks (Kirk \& Schneider 1987). In the following calculation, we will assume the spectrum to be power-law with exponential cutoffs above $E_{\max }$ and below $E_{\min }$.

Given that both $E_{i, \max }$ and $E_{i, \text { min }}$ are rigidity dependent (i.e. $E_{i, \max (\min )} \propto Z_{i} E_{\mathrm{H}, \max (\min )}$ ), the total mass of nuclei of species $i$ released into the intergalactic space by one hypernova is

$$
M_{i, \mathrm{CR}}=A_{i} \int_{E_{i, \min }}^{E_{i, \max }} \frac{d N_{i}}{d E_{i}} d E_{i} \propto \frac{A_{i}}{Z_{i}} U_{i}
$$

3 The value obtained by Katz et al. (2009) is based on pureproton composition assumption. Since iron nuclei attenuation length around $10^{20} \mathrm{eV}$ is similar to that of proton, we adopt this value as the energy production rate in pure heavy nuclei scenario.

${ }^{4}$ It has been suggested that hypernova produces a kinetic energy distribution spreading over a range of velocity as $E_{k} \propto(\Gamma \beta)^{-\alpha}$ (e.g. Soderberg et al. 2006). The final injected CR spectrum from the hypernova should be contributed by different velocity ejecta and the superimposed spectrum becomes softer(Wang et al. 2007). We here consider the highest energy cosmic rays, and for simplicity, just focus on the fastest (semi-relativistic) part of the ejecta and assume $p \simeq 2$ in the following calculation. 
Approximating $Z_{i}=A_{i} / 2$ (except for $\mathrm{H}$ ), and assuming that the same proportions of particles of each species get accelerated (i.e. the value of $M_{i, \mathrm{CR}} / M_{i}$ is independent of $i$ ), we have

$$
U_{\mathrm{H}}: U_{i}: U_{j}=2 M_{\mathrm{H}}: M_{i}: M_{j} . \quad(i, j \neq H, i \neq j)
$$

With the above equation, one can transform the abundances of nuclei of each species to the abundances of these nuclei at certain given energy, as described by Eq.(2).

In the hypernova scenario, cosmic ray particles originate from the circum-stellar wind material or hypernova ejecta material. The WR stellar wind contains abundant intermediate mass elements such as $\mathrm{C}$ and $\mathrm{O}$, which dominate the chemical enrichment of interstellar medium (ISM) (e.g. Abbott 1982; Bieging 1990). The mass ratio of different elements for typical WR stellar wind is $M_{\mathrm{He}}: M_{\mathrm{C}}: M_{\mathrm{O}}: M_{\mathrm{X}}=0.32: 0.39: 0.25: 0.04$ (Bieging 1990), derived based on observation of emission line of these elements in WR stars and the stellar evolution model (see e.g. Willis 1982; Prantzos et al. 1986; van der Hucht et al. 1986, where X denotes the elements other than $\mathrm{He}, \mathrm{C}$ and $\mathrm{O}$. In the following calculation, we will use this composition as a representative case for the cosmic rays from the stellar wind.

On the other hand, the hypernova ejecta contains both intermediate mass elements and heavy elements. It consists of products of nuclear reaction in the interior of the WR star, such as $\mathrm{C}, \mathrm{O}, \mathrm{Mg}, \mathrm{Fe}$, as well as some heavier elements produced by the explosive nucleosynthesis. Chemical composition of the ejecta can be derived by modeling the spectra and light curves of hypernovae (e.g. Nakamura et al. 2001; Fryer et al. 2006) or through numerical simulation of SN explosion based on some specific stellar model (e.g. Georgy et al. 2009). Best fit for the early spectra and light curve of SN 1998bw is obtained in the model named "CO138E50" by Nakamura et al. (2001), where the input explosion kinetic energy and ejecta mass are, respectively, $E_{K}=5 \times 10^{52} \mathrm{ergs}$ and $M_{\mathrm{ej}}=10 M_{\odot}$. This model yields a chemical composition ${ }^{5}$ of $M_{\mathrm{C}}: M_{\mathrm{O}}: M_{\mathrm{Ne}}: M_{\mathrm{Mg}}: M_{\mathrm{Si}}: M_{\mathrm{S}}: M_{\mathrm{Ca}}: M_{\mathrm{Fe}}=$ $0.006: 0.71: 0.037: 0.034: 0.083: 0.041: 0.007: 0.09$. In the following calculation, we will use this composition as a representative case for cosmic rays from the hypernova ejecta. In both scenarios, the amount of hydrogen is negligible, because the progenitor of Type $\mathrm{Ib} / \mathrm{c} \mathrm{SN}$ is stripped of $\mathrm{H}$ envelope, as indicated by lack of $\mathrm{H}$ line in the SN spectrum (Iwamoto et al. 1998; Nakamura et al. 2001). So during the explosion stage, there is little amount of $\mathrm{H}$ in both the wind and the SN ejecta.

\section{THE PROPAGATED SPECTRUM AND CHEMICAL} COMPOSITION

\subsection{Propagation of UHE CR nuclei}

While UHECRs are propagating in the intergalactic space, there are three main attenuation processes due to interaction with CMB or CIB photons: the photodisintegration process, the Bethe-Heitler process and the photopion production process. The photodisintegration process causes nuclei to lose their nucleons and thus change

\footnotetext{
5 We neglect the elements with mass less than $10^{-3} M_{\odot}$ and treat elements heavier than $\mathrm{Fe}$ (e.g. Ni, Co) as Fe because such elements would decay into Fe finally.
}

their species, but does not reduce their Lorentz factor, while the latter two processes can reduce the Lorentz factors of nuclei. Thus, we need to consider the evolution of the mass number $A(t)$ and Lorentz factor $\gamma_{N}(t)$ of a nucleus with time jointly. In the hypernovae scenario, given that $E_{\max } / A \lesssim 10^{19} \mathrm{eV}$, the corresponding maximum energy of each nucleon is below the threshold energy for photopion production with CMB photons even at high redshifts. Although these nuclei can interact with more energetic CIB photons, the photopion energy loss rate with such photons is much lower than the energy loss via the Bethe-Heitler process by interacting with much denser CMB photons, so we neglect the photopion energy loss in the following calculation. In this work, we adopt the CIB model suggested by Razzaque et al. (2009) and Finke et al. (2010), and use the online data of the CIB intensity at different redshifts ${ }^{6}$.

When an UHE nucleus is propagating in the intergalactic space, its Lorentz factor evolves with time as

$$
-\frac{d \gamma_{N}(t)}{d t}=\gamma_{N}(t) H(z)+\dot{\gamma}_{N, \mathrm{BH}}(t)
$$

where $\gamma_{N}(t) H(z)$ represents the adiabatic energy losses due to cosmological expansion and $\dot{\gamma}_{N, \mathrm{BH}}(t)$ is energy loss rate due to the Bethe-Heitler process. Here $H(z)=$ $H_{0} \sqrt{\Omega_{m}(1+z)^{3}+\Omega_{\Lambda}}$ is the Hubble constant at time $t=t(z)$, where $H_{0}=71 \mathrm{kms}^{-1} \mathrm{Mpc}^{-1}, \Omega_{m}=0.27$ and $\Omega_{\Lambda}=0.73$. In an isotropic photon background, the Bethe-Heitler energy loss rate of a nucleus of the Lorentz factor $\gamma_{i}$ is given by

$$
\begin{aligned}
\dot{\gamma}_{N, \mathrm{BH}}(t) & =\frac{Z^{2}}{A} \dot{\gamma}_{p, \mathrm{BH}}(t) \\
& =\frac{Z^{2}}{A} \frac{c}{2 \gamma_{N}} \int_{\epsilon_{\mathrm{th}}}^{\infty} d \epsilon_{\gamma} \sigma_{\mathrm{BH}}\left(\epsilon_{\gamma}\right) f_{\mathrm{BH}}\left(\epsilon_{\gamma}\right) \epsilon_{\gamma} \int_{\epsilon_{\gamma} / 2 \gamma_{i}}^{\infty} d \epsilon \frac{n_{\gamma}(\epsilon, z)}{\epsilon^{2}}
\end{aligned}
$$

where $\dot{\gamma}_{p, \mathrm{BH}}(t)$ is the Bethe-Heitler energy loss rate for a proton of the same Lorentz factor $\gamma_{N}, Z$ is the nuclear charge of the nucleus, $\sigma_{\mathrm{BH}}$ and $f_{\mathrm{BH}}$ are the cross section and the fraction of energy loss in one interaction for the Beth-Heitler process (Chodorowski et al. 1992), and $\epsilon_{\mathrm{th}}$ is the threshold energy for the interaction. Here $\epsilon_{\gamma}$ is the photon energy in the rest frame of the nucleus while $\epsilon$ is the photon energy in the lab frame. $n_{\gamma}(\epsilon, z)$ is the number density of background $(\mathrm{CMB}+\mathrm{CIB})$ photons of energy $\epsilon$ at redshift $z$.

The nucleus suffers from loss of nucleons by the photodisintegration process, so the mass number evolves as

$$
-\frac{d A}{d t}=R_{A}\left(t, \gamma_{i}\right)
$$

where $R_{A}$ is the reaction rate, which is

$$
R_{A}\left(t, \gamma_{N}\right)=\frac{c}{2 \gamma_{N}^{2}} \int_{\epsilon_{\mathrm{th}}}^{\infty} d \epsilon \sigma_{\mathrm{dis}, A}(\epsilon) \epsilon \int_{\epsilon / 2 \gamma_{i}}^{\infty} d \epsilon_{\gamma} \frac{n_{\gamma}\left(\epsilon_{\gamma}, z\right)}{\epsilon_{\gamma}^{2}},
$$

where $\sigma_{\mathrm{dis}, A}$ is the total photo-disintegration cross section for a nucleus of mass number $A$. The photodisintegration cross section is dominated by the giant

\footnotetext{
${ }^{6}$ http://www.phy.ohiou.edu/ finke/EBL/index.html
} 
dipole resonance (GDR) up to $30 \mathrm{MeV}$, with the threshold energy between about $10 \mathrm{MeV}$ and $20 \mathrm{MeV}$ for all nuclei (in the nucleus rest frame). The cross section in this energy range can be modeled by a Gaussian form (e.g. Puget et al. 1976; Stecker \& Salamon 1999; Hooper et al. 2008) or a Lorentzian form (e.g. Khan et al. 2005; Anchordoqui et al. 2007; Hooper et al. 2007). From 30MeV to $150 \mathrm{MeV}$, the quasi-deuteron process becomes dominant and the cross section can be approximated as a plateau (e.g. Puget et al. 1976; Stecker \& Salamon 1999; Ahlers \& Taylor 2010). In this work, we use the tabulated cross section data generated by the code TALYS ${ }^{7}$ (Goriely et al. 2008) which considered all the individual nucleon emission channels for nuclei with $A>5$. For nuclei with $A<5$, we adopt the Gaussian form near the threshold along with a plateau at higher energies to describe the total cross section.

We use the Runge-Kutta method to solve Eq. 5 and Eq. 7 jointly so that we can trace the evolution history of the Lorentz factor $\gamma_{N}\left(z ; z_{s}, E_{s}, A_{s}\right)$ and mass number $A\left(z ; z_{s}, E_{s}, A_{s}\right)$ of a specific nucleus injected from the source at redshift $z=z_{s}$, where $E_{s}$ and $A_{s}$ are the initial energy and mass number of the nucleus. The evolution of the mass number is assumed to develop along the PugetStecker-Bredekamp chain (see e.g. Puget et al. 1976; Stecker \& Salamon 1999; Ahlers \& Taylor 2010). We also record the redshifts, Lorentz factors and number of secondary protons ${ }^{8}$ that are disintegrated from the parent nuclei so that the contribution of secondary protons to cosmic-ray spectrum can be properly included. The nuclei are injected from the maximum redshift $z_{\max }=6$ to the minimum redshift $z_{\min }=0.001$ (corresponding to a distance $D=4 \mathrm{Mpc}$ ). The number density of hypernovae at redshift $z$ is assumed to follow the star formation history (SFH), given by (Hopkins \& Beacom 2006; Yüksel et al. 2008)

$$
n(z) \propto\left\{\begin{array}{l}
(1+z)^{3.4}, z<1 \\
(1+z)^{-0.3}, 1<z<4 \\
(1+z)^{-3.5}, z>4 .
\end{array}\right.
$$

We collect all the nuclei and secondary products that arrive at the Earth $(z=0)$ and put them into the corresponding energy bin and species bin according to their Lorentz factors $\gamma(z=0)$ and mass numbers $A(z=0)$. Then we can get the energy spectrum of cosmic rays in each species bin, or get the all-particle energy spectrum by adding up all species particles at a certain energy.

\subsection{The final spectrum and composition}

The cosmic ray composition at the sources strongly affect the final composition and energy spectrum of cosmic rays arriving at the Earth. First, we consider the chemical composition at the source equal to that of the WR stellar wind, where the mass ratio among dominant elements is $M_{\mathrm{He}}: M_{\mathrm{C}}: M_{\mathrm{O}}=0.32: 0.39: 0.25$ with negligible $\mathrm{H}$. The initial spectrum at the source is assumed to be a power law with $p=2$. The result of the final spectrum and composition are presented in Fig. 1. One can

\footnotetext{
7 http://www.talys.edu/

8 Since the neutrons emitted by the photodisintegration process will decay into protons very soon compared to the propagation time, we just treat the neutrons emitted as protons of the same energy in the calculation.
}

see that with the typical WR stellar wind composition, the cosmic ray flux drops too fast at high energies to account for the observations by PAO. The apparent reason for the fast drop is that the low $E_{\max }$ for intermediate mass nuclei such as $\mathrm{C}$ and $\mathrm{O}$ results in an exponentially cutoff at energy $\lesssim 10^{20} \mathrm{eV}$. However, the situation will not be ameliorated even if we raise $E_{\max , O}$ to higher energies, e.g. to $3 \times 10^{20} \mathrm{eV}$. This is because that lower energy background photons, which are more abundant, are involved in the photodisintegration interaction for intermediate mass nuclei due to their higher Lorentz factors, compared with heavy nuclei of the same energy. Therefore the attenuation lengths for the intermediate nuclei with energies larger than $10^{20} \mathrm{eV}$ are so small (e.g. Puget et al. 1976; Allard et al. 2006) that even those who emitted by the nearest sources at $z_{\min }=0.001$ (i.e. $4 \mathrm{Mpc}$ ) will be effectively attenuated by the background photons and hence the flux of cosmic rays at such energies is severely suppressed after propagation. Only if $E_{\max , \mathrm{O}}$ is extremely high (e.g. $\sim 3 \times 10^{21} \mathrm{eV}$ ) so that secondary protons of energies up to $2-3 \times 10^{20} \mathrm{eV}$ can be produced in photodisintegration process, and then the spectrum becomes flat at the highest energy end (Allard et al. 2007). However, such a high $E_{\max , \mathrm{O}}$ can hardly be achieved in the hypernova scenario.

Presence of heavier nuclei in the source composition would be beneficial to fit the observation data, since heavy nuclei have higher steepening energies due to interactions with background photons. Hypernova ejecta produced after the explosion can provide heavy elements besides intermediate mass elements. Fig. 2 shows the propagated spectrum for cosmic ray composition at the sources equal to the hypernova ejecta composition in the "CO138E50" model for SN 1998bw (Nakamura et al. 2001). With the contribution by heavy nuclei at the sources, the propagated spectrum is in better agreement with the PAO data than the wind composition case.

The fit can be further improved if some non-standard effects are taken into account. In panel a of Fig. 3, we increase the initial iron abundance by a factor of 3, i.e. iron nuclei constitute a fraction of $\sim 25 \%$ of the total mass at the sources. This could be achieved since the enrichment of heavy elements is not uniformly distributed spatially, as already seen in some Galactic supernova remnants (e.g. Hwang et al. 2000). A larger explosion energy would also lead to more heavy elements synthesized during the explosion (e.g. Metzger et al. 2011). As iron nuclei have larger steepening energy in the spectrum due to interactions with background photons, a harder spectrum is expected at the highest energy if more iron nuclei are present. This is consistent with earlier results in Allard et al. (2008). Another possible effect is a hard injection CR spectrum. Although in conventional shock acceleration theory, the power-law index is $p \simeq 2$, some observations (e.g. Horns \& Aharonian 2004; Green 2009; Reynolds et al. 2011; Abdo et al. 2011) and theoretical calculations (e.g. Ellison et al. 1996; Malkov 1997; Vainio \& Schlickeiser 1999; Vainio et al. 2003; Tammi \& Duffy 2009) have suggested harder cosmic-ray spectra with indices $p<2$. In panel $b$, we show the propagated spectrum for an injection spectrum with power-law index $p=1.6$. One can see that the fit to the data gets better than the case of $p=2$. Besides, the source density distribu- 
tion in the nearby universe could be nonuniform. Since our Galaxy locates inside the Local group and Local supercluster, we examine the effect of local overdensity of sources on the propagated spectrum. Panel $c$ shows the spectrum for a local overdensity in the source number by a factor of 2 relative to the average within the size of $30 \mathrm{Mpc}$ (Blanton et al. 2001). Due to the attenuation by background photons, higher energy cosmic rays are mainly contributed by closer sources. Thus a local excess of the source number density increases the flux of higher energy cosmic rays and thus hardens the propagated spectrum at the highest energy end.

We should note that, although the deviation between the propagated spectrum and observational data is relatively large in the wind composition scenario, the situation can also be improved if some of the above effects are taken into account. Panel d of Fig. 3 presents the result after considering a harder injection cosmic-ray spectrum of $p=-1.6$ as well as a local overdensity in the source number by a factor of 2 . The theoretical energy spectrum agrees reasonably well with the observational data.

In Fig. 4, we present how the average mass number of cosmic rays evolves with energy for different scenarios discussed above. Note that the starting energy of this figure is set to $3 \times 10^{18} \mathrm{eV}$ since our model do not account for the CR spectrum data below this energy, for which a Galactic component contribution is needed. One can see that, for the hypernova ejecta composition scenarios, the average mass number increases gradually with energy, which is consistent with the finding by PAO that the composition of UHECRs becomes increasingly heavy with energy. In the wind composition scenarios, the average mass number increases more slowly with energy. However, since the hadronic interaction models at such high energies are not well understood, such a composition is still consistent with the measurements within the uncertainties of theoretical expectations.

\section{DISCUSSIONS AND CONCLUSIONS}

We have shown that, with some fraction of heavy nuclei such as iron in the source composition, the semirelativistic hypernova model can explain the energy spectrum and composition of ultra-high energy cosmic rays as measured by PAO. The heavy elements may originate from the hypernova ejecta and are brought into the forward shock region via the Rayleigh-Taylor (R-T) instability. R-T fingers are seen to be able to reach the forward shock front or overtake the forward shocks (e.g. Jun et al. 1996; Blondin \& Ellison 2001; Blondin et al. 2001; Rakowski et al. 2011), so they should be able to bring heavy elements into the ambient medium. Observations of some Galactic supernova remnants such as Cas A and SN 1006, as well as SN 1987A in Large Magellanic Cloud, have provided direct and indirect evidences for protrusion of heavy elements in the material beyond the blast wave (e.g. Erickson et al. 1988; Fesen \& Gunderson 1996; Hwang et al. 1998; Hughes et al. 2000).
When the ejecta is being decelerated by the sweptup ambient wind material, reverse shocks are also forming. The reverse shock may accelerate particles in the shocked ejecta, which provides an alternative site where UHE heavy nuclei are accelerated. The reverser shock in the hypernova case is typically mildly relativistic with a speed about $\beta_{\mathrm{rs}} \simeq 0.5$, as shown in the appendix. Since the internal energy density in the shocked ejecta and shocked wind are equal, the magnetic field in the reverse shock is $B_{\mathrm{rs}}=\left(\epsilon_{B \mathrm{r}} / \epsilon_{B}\right)^{1 / 2} B$, where $\epsilon_{B \mathrm{r}}$ is the magnetic field energy equipartition factor in reverse shock and $\epsilon_{B}$ is the same factor for the forward shock. Then we obtain the maximum energy of particles accelerated by the reverse shock

$$
\begin{aligned}
E_{\mathrm{max}, \mathrm{rs}} & =Z e B_{\mathrm{rs}} R_{\mathrm{HN}} \beta_{\mathrm{rs}} \\
& =1.8 \times 10^{20}\left(\frac{Z}{26}\right) \epsilon_{B \mathrm{r},-1}^{1 / 2}\left(\frac{\beta_{\mathrm{sh}} \beta_{\mathrm{rs}}}{0.5}\right) \dot{M}_{-5}^{1 / 2} v_{w, 3}^{-1 / 2} \mathrm{eV},
\end{aligned}
$$

which can also reach ultra-high energies for heavy nuclei.

In summary, hypernovae remnants are shown to be potential sources of UHECRs. They have been discovered to be present within the GZK horizon, $100 \mathrm{Mpc}$. Due to the semi-relativistic ejecta produced by the explosion, they can accelerate particles to ultra-high energies. Hypernovae naturally provide intermediate mass or heavy elements either in the form of stellar wind or the hypernova ejecta itself. In this paper, we have calculated the propagated spectrum and composition of UHECRs arriving at the Earth given an initial cosmic ray spectrum and composition at the sources produced by hyerpnovae. We find that with the source cosmic-ray composition equal to that of the hypernova ejecta, the propagated energy spectrum and composition are compatible with the measurements by PAO.

There are suggestions that past Galactic hypernova could contribute to cosmic rays in the energy range above the knee (Budnik et al. 2008) or even UHECRs (Calvez et al. 2010). However, the event rate of such Galactic hypernovae is largely unknown. There has been evidence indicating that hypernovae associated with sub-energetic GRBs are preferentially found in low-metallicity galaxies (e.g. Woosley \& Bloom 2006; Stanek et al. 2006), which would imply that hypernova rates in normal metallicity galaxies such as our Milky Way may be low.

We are grateful to the anonymous referee for the valuable report, and to A. M. Taylor, P. Mészáros, S. Razzaque and Z. G. Dai for useful discussions. This work is supported by the NSFC under grants 10973008 and 11033002, the 973 program under grant 2009CB824800, the program of NCET, and the Fok Ying Tung Education Foundation

\section{APPENDIX}

\section{THE SPEED OF REVERSE SHOCKS}

When the ejecta is being decelerated by the swept-up ambient wind material, reverse shocks are also forming. The shocked wind and shocked ejecta are in pressure balance and separated by the contact discontinuity. The semirelativistic ejecta get decelerated at $R_{\mathrm{HN}} \simeq 5 \times 10^{16} E_{k, 51}\left(\Gamma_{0} / 2\right)^{-2} \dot{M}_{-5}^{-1} v_{w, 3} \mathrm{~cm}$, where $E_{k}$ and $\Gamma_{0}$ are, respectively, the 
total kinetic energy and the initial Lorentz factor of the semi-relativistic ejecta. Since the shock expansion is expected to be adiabatic, we have

$$
E_{\mathrm{sh}, \mathrm{w}}+E_{\mathrm{sh}, \mathrm{ej}}=E_{k},
$$

where $E_{\mathrm{sh}, \mathrm{w}} \simeq 4 / 3 \pi \sigma \beta^{2} \Gamma^{2} R^{3} \rho_{w}(R) c^{2}$ (Blandford \& McKee 1976) is the total energy transferred to the shocked wind from the blast wave, $E_{\mathrm{sh}, \mathrm{ej}} \simeq \Gamma^{2}\left(e^{\prime}+\rho^{\prime} c^{2}+\beta^{2} p^{\prime}\right) V_{\mathrm{sh}, \mathrm{ej}}$ is the total energy in the shocked ejecta, $\Gamma$ is the Lorentz factor of the shocked material and $\rho_{w}(R)$ is the wind density at radius $R$. Here $\sigma$ is a coefficient with $\sigma \rightarrow 0.35$ when $\beta \rightarrow 1$ and $\sigma \rightarrow 0.73$ when $\beta \rightarrow 0$. We use $\sigma=0.73-0.38 \beta$ to bridge the gap between the non-relativistic case and ultra-relativistic case, as used in Huang et al. (1998). $e^{\prime}=\frac{\hat{\gamma} \gamma_{\mathrm{rs}}+1}{\hat{\gamma}-1}\left(\gamma_{\mathrm{rs}}-1\right) \rho_{\mathrm{ej}} c^{2}, \rho^{\prime}=\frac{\hat{\gamma} \gamma_{\mathrm{rs}}+1}{\hat{\gamma}-1} \rho_{\mathrm{ej}}$ and $p^{\prime}=(\hat{\gamma}-1) e^{\prime}$ are respectively, the internal energy density, rest mass density and pressure in the comoving frame of shocked material, and $\hat{\gamma}$ is the adiabatic index. $V_{\mathrm{sh}, \mathrm{ej}}=V_{0} /\left(\frac{\hat{\gamma} \gamma_{\mathrm{rs}}+1}{\hat{\gamma}-1}\right)$ is the volume of shocked ejecta, where $V_{0}$ is the volume of the ejecta before being shocked. Here $\gamma_{\mathrm{rs}}$ is the Lorentz factor of the reverse shock, which relates with the Lorentz factor $\Gamma$ of the shocked material by $\gamma_{\mathrm{rs}} \simeq \Gamma_{0} \Gamma\left(1-\beta_{0} \beta_{\mathrm{se}}\right)$, where $\beta_{0}$ and $\beta_{\mathrm{se}}$ are the velocities of the unshocked ejecta and shocked ejecta respectively. Solving Eq. (A1) with typical values for the parameters, we finally get the Lorentz factor and speed of the reverse shock,

$$
\gamma \approx 1.15, \beta_{\mathrm{rs}}=\sqrt{1-1 / \gamma^{2}} \approx 0.5
$$

\section{REFERENCES}

Abbasi, R. U., Abu-Zayyad, T., Amann, J. F., et al. 2004, Physical Review Letters, 92, 151101

Abbott, D. C. 1982, ApJ, 263, 723

Abdo, A. A., et al. 2011, ApJ, 734, 28

Abraham, J., et al. 2010, Physical Review Letters, 104, 091101

Ahlers, M., \& Taylor, A. M. 2010, Phys. Rev. D, 82, 123005

Allard, D., Ave, M., Busca, N., Malkan, M. A., Olinto, A. V., Parizot, E., Stecker, F. W., \& Yamamoto, T. 2006, JCAP, 9, 5

Allard, D., Parizot, E., \& Olinto, A. V. 2007, Astroparticle Physics, 27, 61

Allard, D., Busca, N. G., Decerprit, G., Olinto, A. V., \& Parizot, E. 2008, jcap, 10,33

Allard, D. 2009, arXiv:0906.3156

Aloisio, R. 2011, arXiv:1104.0329

Anchordoqui, L. A., Beacom, J. F., Goldberg, H., Palomares-Ruiz, S., \& Weiler, T. J. 2007, Physical Review Letters, 98, 121101

Berezinsky, V., Gazizov, A., \& Grigorieva, S. 2006, Phys. Rev. D, 74,043005

Bieging, J. H. 1990, The Evolution of the Interstellar Medium, 12, 137

Biermann, P. L., \& Strittmatter, P. A. 1987, ApJ, 322, 643

Blandford, R. D., \& McKee, C. F. 1976, Physics of Fluids, 19, 1130

Blanton, M., Blasi, P., \& Olinto, A. V. 2001, Astroparticle Physics, 15, 275

Blondin, J. M., \& Ellison, D. C. 2001, ApJ, 560, 244

Blondin, J. M., Borkowski, K. J., \& Reynolds, S. P. 2001, ApJ, 557,782

R. Budnik, B. Katz, A. MacFadyen, E. Waxman, 2008, ApJ, 673, 928

Calvez, A., Kusenko, A., \& Nagataki, S. 2010, Physical Review Letters, 105, 091101

Campana, S., et al. 2006, Nature, 442, 1008

Chakraborti, S., Ray, A., Soderberg, A. M., Loeb, A., \& Chandra, P. 2011, Nature Communications, 2,

Chevalier, R. A., \& Li, Z.-Y. 1999, ApJ, 520, L29

Chodorowski, M. J., Zdziarski, A. A., \& Sikora, M. 1992, ApJ, 400, 181

Crowther P. A. 2007, ARA\&A, 45, 177

Dermer, C. D., \& Atoyan, A. 2006, New Journal of Physics, 8, 122

Ellison, D. C., Baring, M. G., \& Jones, F. C. 1996, ApJ, 473, 1029

Erickson, E. F., Haas, M. R., Colgan, S. W. J., et al. 1988, ApJ, 330, L39

Fesen, R. A., \& Gunderson, K. S. 1996, ApJ, 470, 967

Finke, J. D., Razzaque, S., \& Dermer, C. D. 2010, ApJ, 712, 238

Fryer, C. L., Young, P. A., \& Hungerford, A. L. 2006, ApJ, 650, 1028

Galama, T. J., et al. 1998, Nature, 395, 670

Georgy, C., Meynet, G., Walder, R., Folini, D., \& Maeder, A. 2009, A\&A, 502, 611

Green, D. A. 2009, Bulletin of the Astronomical Society of India, 37,45
Goriely, S., Hilaire, S., \& Koning, A. J. 2008, A\&A, 487, 767

Guetta, D., \& Della Valle, M. 2007, ApJ, 657, L73

Hooper, D., Sarkar, S., \& Taylor, A. M. 2007, Astroparticle Physics, 27, 199

Hooper, D., Sarkar, S., \& Taylor, A. M. 2008, Phys. Rev. D, 77, 103007

Hopkins, A. M., \& Beacom, J. F. 2006, ApJ, 651, 142

Horns, D., \& Aharonian, F. A. 2004, 5th INTEGRAL Workshop on the INTEGRAL Universe, 552, 439

Huang, Y. F., Dai, Z. G., \& Lu, T. 1998, A\&A, 336, L69

Hughes, J. P., Rakowski, C. E., Burrows, D. N., \& Slane, P. O. 2000, ApJ, 528, L109

Hwang, U., Hughes, J. P., \& Petre, R. 1998, ApJ, 497, 833

Hwang, U., Holt, S. S., \& Petre, R. 2000, ApJ, 537, L119

Iwamoto, K., et al. 1998, Nature, 395, 672

Jun, B.-I., Jones, T. W., \& Norman, M. L. 1996, ApJ, 468, L59

Katz, B., Budnik, R., \& Waxman, E. 2009, JCAP, 3, 20

Khan, E., Goriely, S., Allard, D., Parizot, E., Suomijärvi, T., Koning, A. J., Hilaire, S., \& Duijvestijn, M. C. 2005, Astroparticle Physics, 23, 191

Kirk, J. G., \& Schneider, P. 1987, ApJ, 315, 425

Kulkarni, S. R., et al. 1998, Nature, 395, 663

Liu, R.-Y., Wang, X.-Y., \& Dai, Z.-G. 2011, MNRAS, in press, arXiv:1108.1551

Malkov, M. A. 1997, ApJ, 485, 638

Mazzali, P. A., et al. 2006, Nature, 442, 1018

Metzger, B. D., Giannios, D., \& Horiuchi, S. 2011, MNRAS, 415, 2495

Mirabal, N., Halpern, J. P., An, D., Thorstensen, J. R., \& Terndrup, D. M. 2006, ApJ, 643, L99

Mücke, A., Engel, R., Rachen, J. P., Protheroe, R. J., \& Stanev, T. 2000, Computer Physics Communications, 124, 290

Murase, K., \& Nagataki, S. 2006, Phys. Rev. D, 73, 063002

Murase, K., Ioka, K., Nagataki, S., \& Nakamura, T. 2006, ApJ, 651, L5

Nakamura, T., Mazzali, P. A., Nomoto, K., \& Iwamoto, K. 2001, ApJ, 550, 991

Paczyński, B. 1998, Gamma-Ray Bursts, 4th Hunstville Symposium, 428, 783

Pe'er, A., Murase, K., \& Mészáros, P. 2009, Phys. Rev. D, 80, 123018

Prantzos, N., Doom, C., De Loore, C., \& Arnould, M. 1986, ApJ, 304, 695

Puget, J. L., Stecker, F. W., \& Bredekamp, J. H. 1976, ApJ, 205, 638

Rakowski, C. E., Laming, J. M., Hwang, U., et al. 2011, ApJ, $735, \mathrm{~L} 21$

Ray, A., \& Chakraborti, S. 2011, arXiv:1103.3992

Razzaque, S., Dermer, C. D.; Finke, J. D., ApJ, 697, 483

Reynolds, S. P., Gaensler, B. M., \& Bocchino, F. 2011,

Space Sci. Rev., 61

Soderberg A. M., et al., 2006, Natur, 442, 1014

Soderberg, A. M., et al. 2010, Nature, 463, 513 
Stecker, F. W., \& Salamon, M. H. 1999, ApJ, 512, 521

Stanek K. Z. et al., Acta Astronomica 56, 333 (2006).

Tammi, J., \& Duffy, P. 2009, MNRAS, 393, 1063

Taylor, A. M., Ahlers, M., \& Aharonian, F. A. 2011, arXiv:1107.2055

Tsunesada, Y. for the Telescope Array Collaboration 2011, arXiv:1111.2507

Vainio, R., \& Schlickeiser, R. 1999, A\&A, 343, 303

Vainio, R., Virtanen, J. J. P., \& Schlickeiser, R. 2003, A\&A, 409, 821

van der Hucht, K. A., Cassinelli, J. P., \& Williams, P. M. 1986, A\&A, 168, 111

Vietri, M. 1995, ApJ, 453, 883

Wang, X.-Y., Razzaque, S., Mészáros, P., \& Dai, Z.-G. 2007, Phys. Rev. D, 76, 083009
Wang, X.-Y., Razzaque, S., \& Mészáros, P. 2008, ApJ, 677, 432

Waxman, E. 1995, Physical Review Letters, 75, 386

Waxman, E. 2004, ApJ, 606, 988

Wick, S. D., Dermer, C. D., \& Atoyan, A. 2004, Astroparticle Physics, 21, 125

Willis, A. J. 1982, Wolf-Rayet Stars: Observations, Physics, Evolution, 99, 87

Willis, A. J. 1991, Wolf-Rayet Stars and Interrelations with Other Massive Stars in Galaxies, 143, 265

Woosley, S. E., \& Bloom, J. S. 2006, ARA\&A, 44, 507

Yüksel, H., Kistler, M. D., Beacom, J. F., \& Hopkins, A. M. 2008, ApJ, 683, L5 


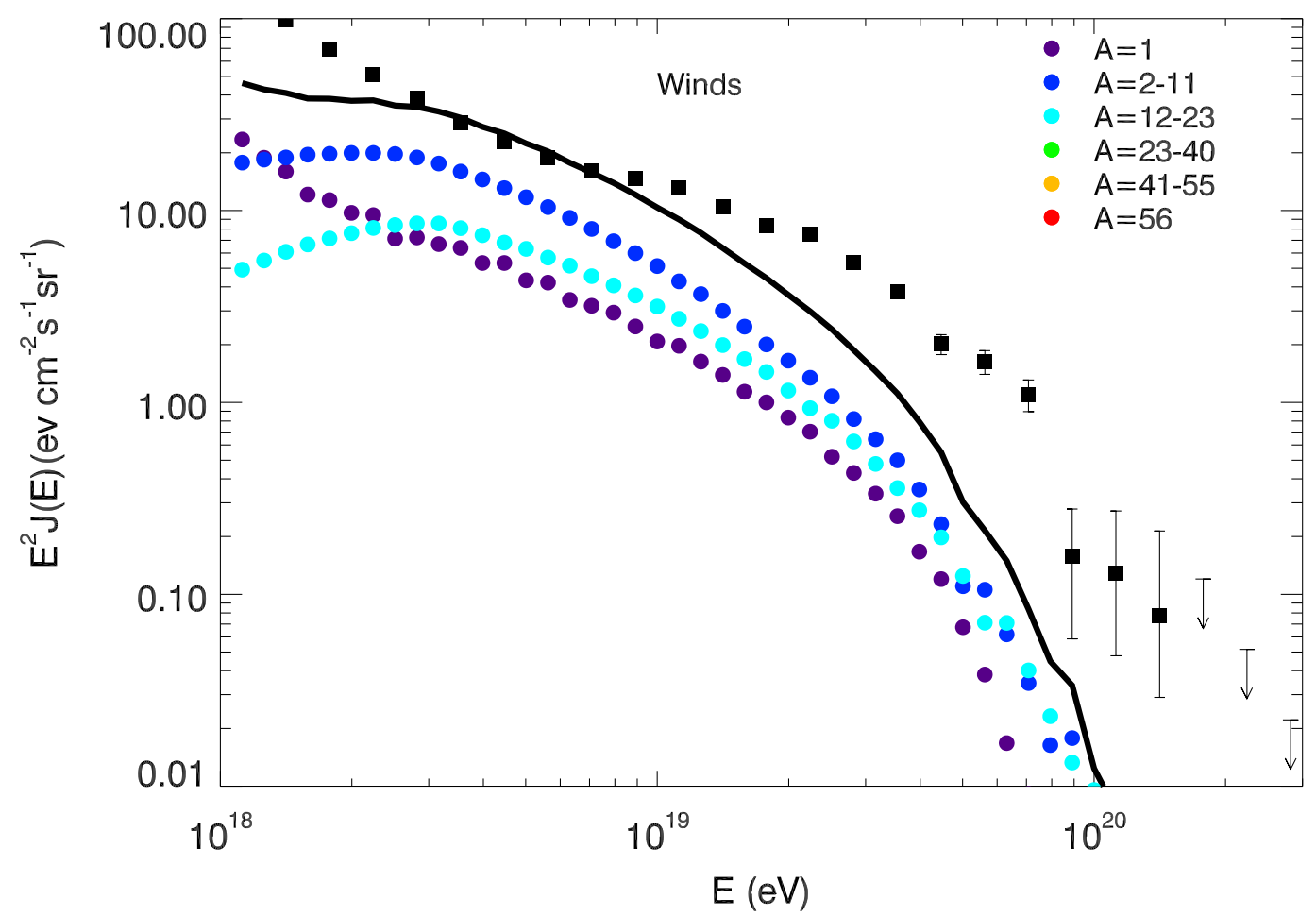

FIG. 1. - The propagated spectrum of cosmic rays assuming a source composition equal to that of the stellar wind of WolfRayet stars, where the mass ratios of dominant elements are He: $\mathrm{C}: \mathrm{O}=0.32: 0.39: 0.25$, and a flat source spectrum with $p=2$ (see text for more details). The black solid line represents the all-particle flux, while other lines represent the contributions by different species as shown in the legend. The black squares are the most recent data measured by PAO, taken from http://www.auger.org/technical_info/ICRC2011/CombinedSpectrum_Auger2011.txt. 


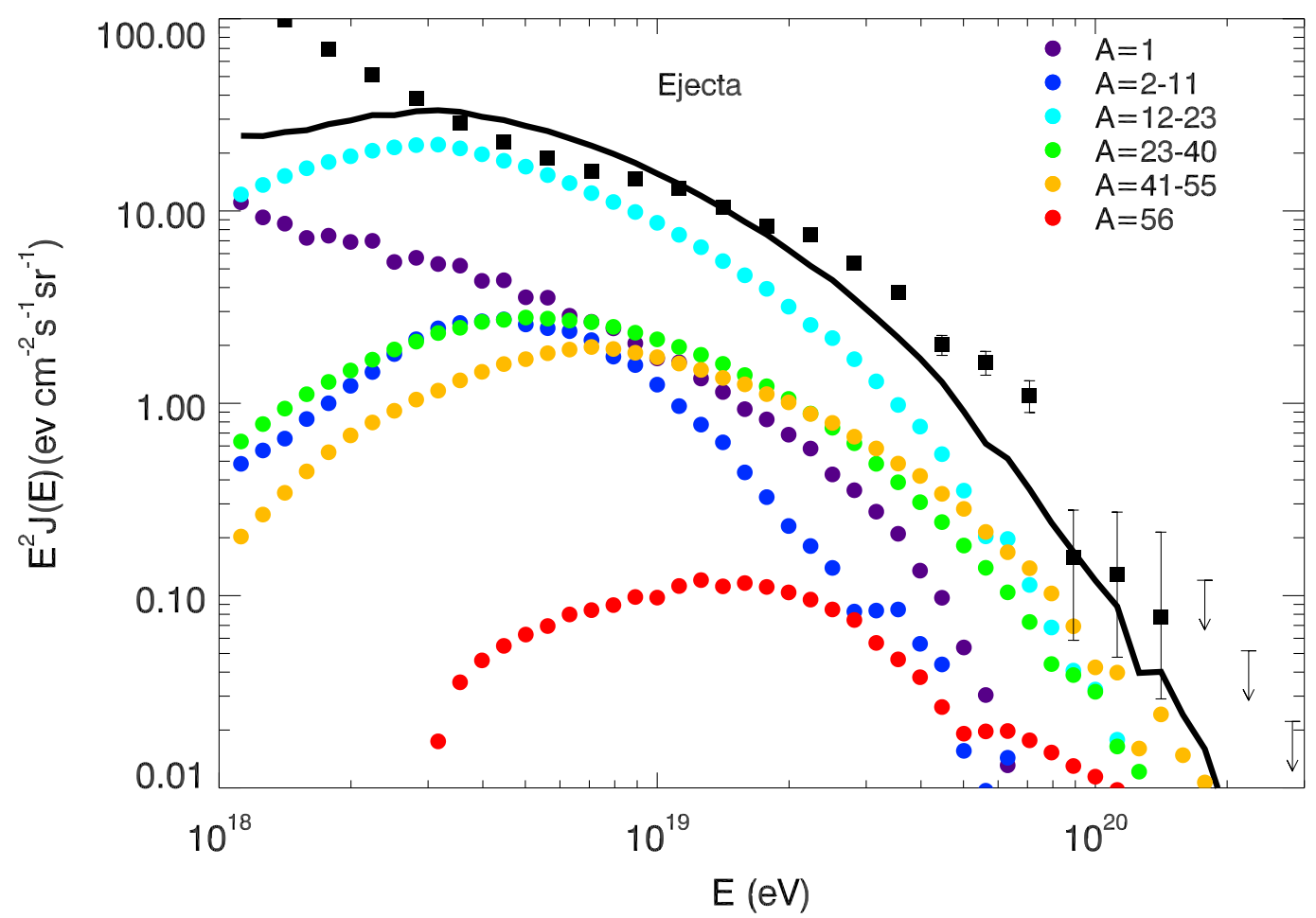

FIG. 2.- Same as Fig.1, but for a source composition equal to that of the hypernova ejecta in the model of SN 1998bw (Nakamura et al. 2001). 

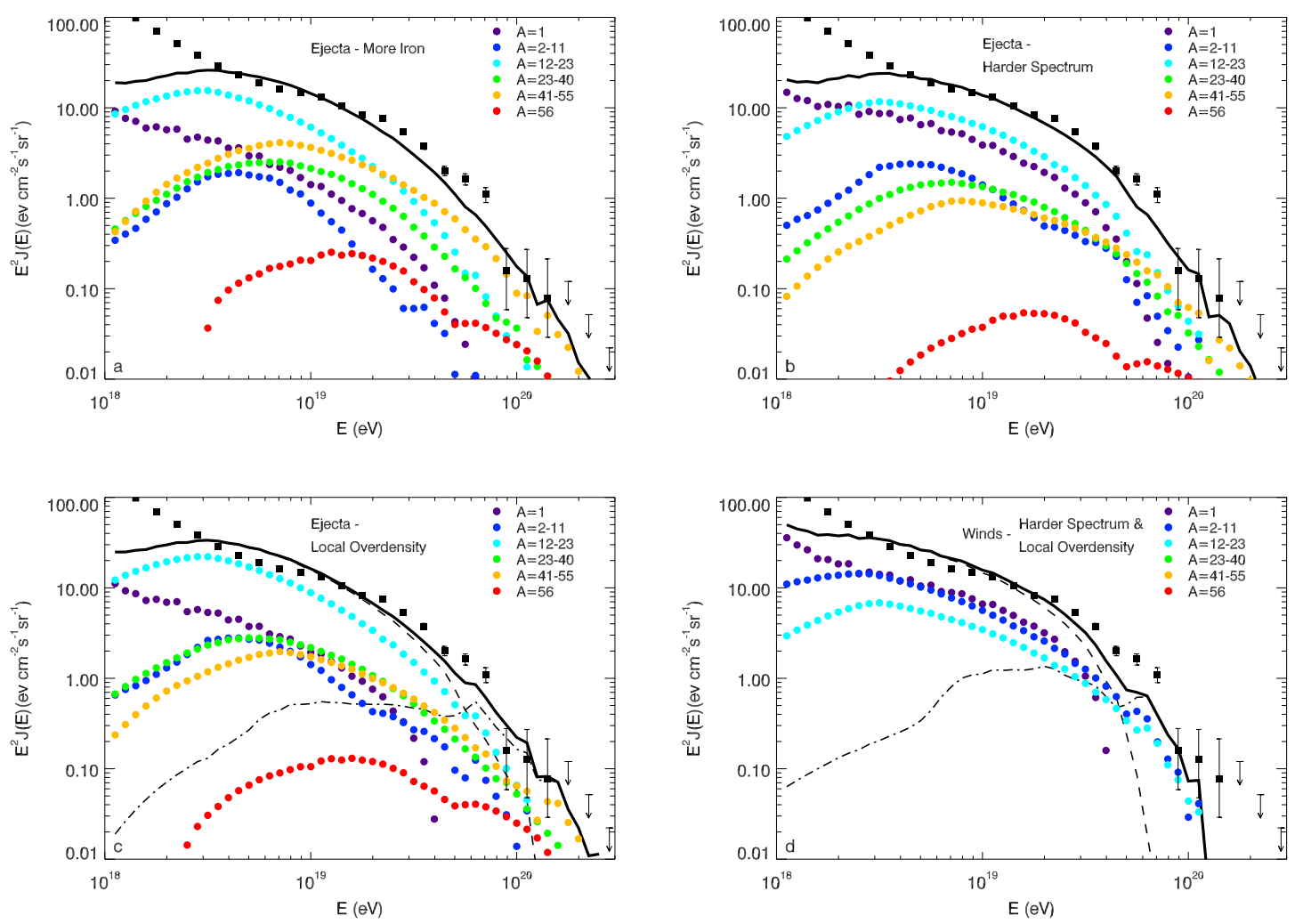

FIG. 3.- Panels $a, b, c$ show the propagated cosmic ray spectrum for the source composition equal to that of the hypernova ejecta, similar to Fig. 2, but with some modifications taken into account. In panel $a$, we increase the abundance of iron nuclei in the ejecta by a factor of 3. In panel $b$, we set a hard initial spectrum with $p=1.6$. In panel $c$, we assume that the local source number density is two times higher than the average within a distance of $30 \mathrm{Mpc}$. The dashed line in panel c represents the contribution by sources beyond $30 \mathrm{Mpc}$ while the dash-dotted line represents the contribution by local sources within $30 \mathrm{Mpc}$. Panel $d$ shows the propagated cosmic ray spectrum for the source composition equal to that of stellar wind, but with the effects of a hard spectrum and a local overdensity being taken into account. See text for more details. 


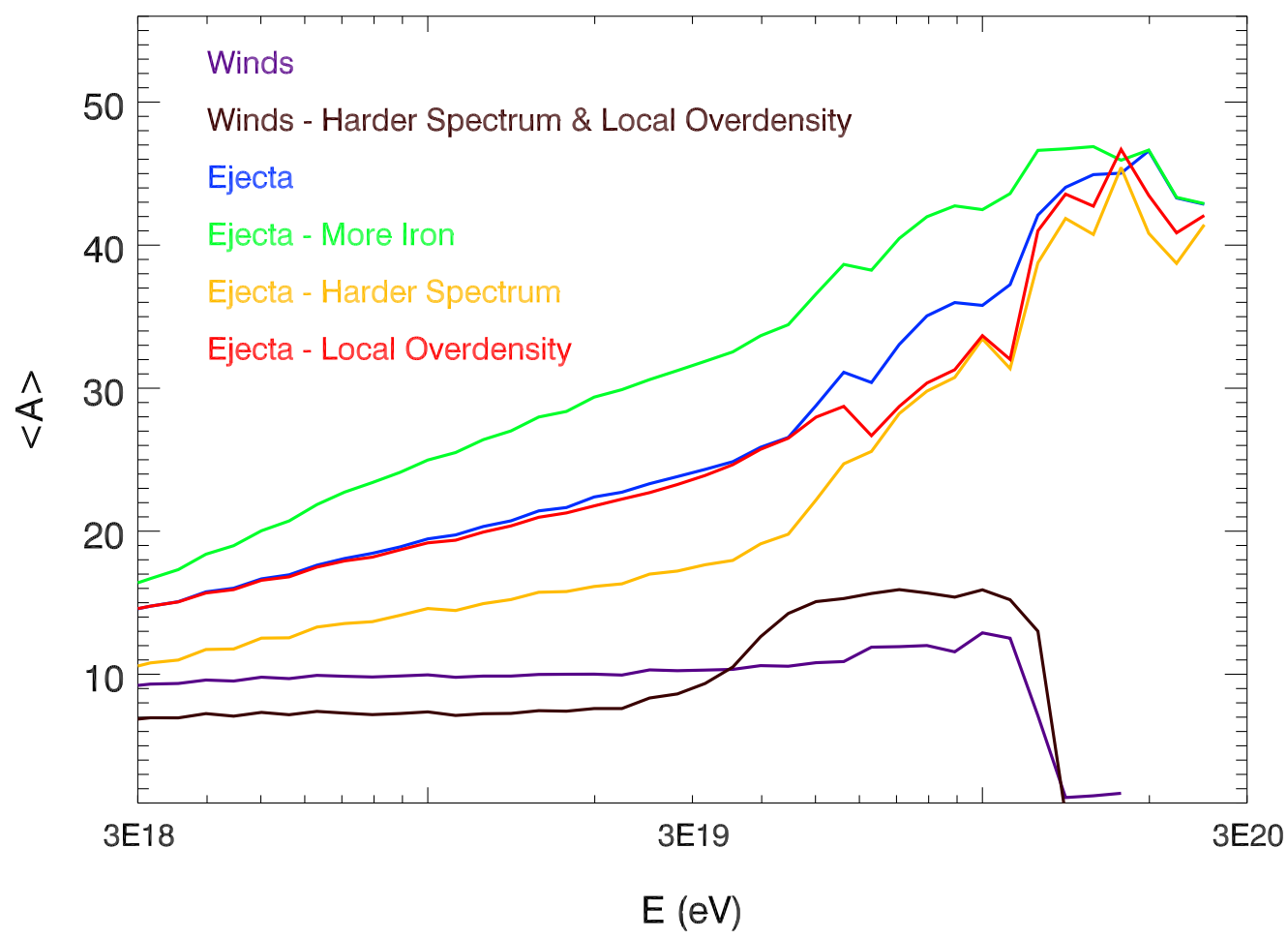

FIG. 4.- The average mass number of propagated cosmic rays arriving at the Earth as a function of the cosmic-ray energy for different cases shown in the above figures. . 